EXTENDED REPORT

\title{
Is manual counting of corneal endothelial cell density in eye banks still acceptable? The French experience
}

\author{
G Thuret, C Manissolle, S Acquart, J-C Le Petit, J Maugery, L Campos-Guyotat, M J Doughty, \\ P Gain
}

See end of article for authors' affiliations .....................

Correspondence to: Gilles Thuret, Service d'ophtalmologie, Pavillon 50 A, Hôpital Bellevue, CHU Saint-Etienne, F-42055 Saint-Etienne Cedex 2, France; gilles.thuret@ univ-st-etienne.fr

Accepted for publication 27 March 2003

\begin{abstract}
Aim: To examine the differences in manual endothelial cell counting methods in French eye banks and to analyse whether these differences could explain some substantial discrepancies observed in endothelial cell density (ECD) for corneas made available for transplant.

Methods: A questionnaire was sent to the 22 eye banks asking for details of the technical features of the light microscopes used, the microscope calibration, strategy for cell counting, the technical staff, and the method of presenting endothelial data.

Results: All eye banks responded and 91\% (20/22) used only manual counting methods, in real time, directly through a microscope, and 62 different technicians, with varying experience, were involved in such counting. Counting of cells within the borders of a grid that were in contact with two adjacent borders was the most common method $(17 / 22,77 \%)$. Of the eight banks $(8 / 22,36 \%)$ that did not calibrate their microscopes, six reported the highest ECD values. Of the 14 others $(64 \%)$, six applied a "magnification correcting factor" to the initial cell counts. In five of these cases, the corrected ECD was lower than estimated on initial count. Most of the banks (12/22, 55\%) counted 100 cells or less in one to six nonadjacent zones of the mosaic. 14 of the banks $(14 / 22,64 \%)$ also graded cell polymegethism while seven $(7 / 22,32 \%)$ also graded pleomorphism ("hexagonality").

Conclusions: Lack of microscope calibration appears to be the leading cause of variance in ECD estimates in French eye banks. Other factors such as differences in counting strategy, the evaluation of smaller numbers of cells, and the different extent of experience of the technicians may also contribute to intraobserver and interobserver variability. Further comparative studies, including cross checking and the outcome of repeated counts from manual methods, are clearly needed with cross calibration to a computer based image archiving and analysis system.
\end{abstract}

A $\mathrm{n}$ assessment of endothelial cell density (ECD) after corneal storage forms the main basis for selection and delivery of corneas by eye banks for transplant purposes. In most eye banks, corneas with an ECD less than $2000 / \mathrm{mm}^{2}$ would not be selected for penetrating keratoplasty. ${ }^{1}$ In France, as elsewhere in Europe, where organ culture is the most common method of corneal storage, ${ }^{1}$ most banks perform endothelial cell (EC) counting manually, under a light microscope. This requires osmotic preparation of the endothelium to dilate the intercellular spaces and make the EC contours visible to the technician. ${ }^{2}$ Counting is generally performed in France through a reticule of known surface area. This method is historically derived from blood cell counts carried out in haematology laboratories using Thoma or Malassez type grids. ${ }^{3}$

Such manual counts might well be considered acceptable. However, an analysis of the data from the 1999-2001 directories of French eye banks ${ }^{4-6}$ indicated some rather substantial differences in the ECD values reported for corneas provided for transplant (Table 1). The mean values for ECD ranged from 1986 to 2848 cells $/ \mathrm{mm}^{2}$ in 1999 , from 2160 to $2732 \mathrm{cell} / \mathrm{mm}^{2}$ in 2000 , and from 2178 to $2795 \mathrm{cell} / \mathrm{mm}^{2}$ in 2001 (data not shown). Such variance cannot be obviously explained by donor age, the nature of the organ culture medium, or by the storage time. Differences in eye bank counting procedures were thought to be a possible cause.

The purpose of this study was to assess whether differences in endothelial assessment could help explain the differences reported and, if appropriate, to identify any procedures that might need correction.

\section{MATERIALS AND METHODS}

In February 2002, a questionnaire was sent to the managers of the 22 eye banks accredited by the French agency for healthcare product safety (Agence Française de Sécurité Sanitaire des Produits de Santé). The questionnaire covered all the materials and methods used for counting-namely, (1) microscope/reticule assembly and its calibration, the microscope features (microscope body, objective, eyepiece), the reticule (design, total and unit surface areas, positioning in the microscope), any calibration used (principle of calibration, frequency, resultant "magnification correcting factor"); (2) counting strategy (viewing area in relation to the corneal centre, number of zones observed, number of reticule units viewed and whether they were adjacent, number of cells assessed); (3) technicians (number and experience); (4) data output (including whether additional morphometry was carried out; and whether there was archiving or transmission of endothelial images). As part of (3), the method of cell counting was assessed in a specific exercise in which the respondents had to mark the cells they would have counted on a very simple schematic mosaic superimposed on a grid (see Results).

\section{Statistical analysis}

The relations between the mean ECD of each eye bank and the explanatory data given by the compilation of the French eye banks directories (mean donor age, mean storage duration, organ culture medium type-that is, Bausch \& Lomb Chauvin-Opsia, Labège, France and/or Eurobio, les Ulys, France) as well as those issued from our questionnaire 
Table 1 Extract from the 7th, 8th, and 9th editions of the directories of the French cornea eye banks (1999, 2000, and 2001 activities)

\begin{tabular}{|c|c|c|c|c|c|c|c|c|}
\hline Eye bank & $\begin{array}{l}\text { Storage medium } \\
\text { manufacturer }\end{array}$ & $\begin{array}{l}\text { Corneas } \\
\text { delivered } \\
\text { (n) }\end{array}$ & $\begin{array}{l}\text { Mean donor } \\
\text { age (years) }\end{array}$ & $\begin{array}{l}\text { Mean storage } \\
\text { time (days) }\end{array}$ & $\begin{array}{l}\text { Mean ECD at } \\
\text { delivery } \\
\left(\text { cells } / \mathrm{mm}^{2} \text { ) }\right.\end{array}$ & $\begin{array}{l}\text { Calibration } \\
\text { (yes/no) }\end{array}$ & $\begin{array}{l}\text { Magnification } \\
\text { correcting factor }\end{array}$ & $\begin{array}{l}\text { Counting strategy } \\
\text { (zone/border) }\end{array}$ \\
\hline 1 & Opsia & 98 & 65 & 20 & 2775 & no & & border \\
\hline 2 & Eurobio & 115 & 65 & 18 & 2763 & no & & zone \\
\hline 3 & Eurobio & 141 & 68 & 14 & 2745 & no & & border \\
\hline 4 & Eurobio & 125 & 60 & 20 & 2690 & no & & other \\
\hline 5 & Opsia & 106 & 70 & 22 & 2646 & no & & zone \\
\hline 6 & Opsia/Eurobio & 67 & 67 & 19 & 2545 & no & & zone \\
\hline 7 & Opsia & 57 & 62 & 22 & 2530 & yes & & border \\
\hline $8^{*}$ & Eurobio & 118 & 55 & 16 & 2520 & yes & $\times 1.5$ & zone \\
\hline 9 & Opsia & 123 & 55 & 13 & 2515 & yes & $\times 0.95$ & zone \\
\hline 10 & Eurobio & 88 & 70 & 20 & 2501 & yes & & zone \\
\hline $11 \dagger$ & Opsia/Eurobio & 412 & 66 & 21 & 2483 & yes & & zone \\
\hline 12 & Opsia/Eurobio & 364 & 65 & 21 & 2439 & yes & $\times 0.8$ & zone \\
\hline 13 & Eurobio & 184 & 63 & 18 & 2427 & yes & $\times 0.9$ & zone \\
\hline 14 & Eurobio & 93 & 56 & 19 & 2345 & no & & zone \\
\hline 15 & Eurobio & 253 & 68 & 23 & 2317 & no & & zone \\
\hline 16 & Opsia/Eurobio & 242 & 67 & 17 & 2277 & yes & & zone \\
\hline $17 \dagger$ & Opsia/Eurobio & 295 & 71 & 19 & 2224 & yes & $\times 0.8$ & zone \\
\hline 18 & NA & 67 & 58 & 15 & 2200 & yes & & zone \\
\hline 19 & Opsia/Eurobio & 187 & 68 & 18 & 2178 & yes & & zone \\
\hline 20 & Opsia & 98 & 66 & 15 & 2158 & $\begin{array}{l}\text { yes } \\
\text { yes }\end{array}$ & $\times 0.64$ & zone \\
\hline 21 & Opsia/Eurobio & 124 & 61 & 16 & 2146 & yes & & other \\
\hline $22 \ddagger$ & Opsia & 46 & 52 & 3 & NA & yes & & zone \\
\hline
\end{tabular}

Figures represented the weighted means of 3 years of activities. The 22 eye banks were classified in descending order of the endothelial cell density (ECD) at delivery. These data underline the disparity of about $25 \%$ of ECDs at delivery. Neither the donor age nor the storage duration correlated with the ECD (respectively $r=-0.04, p=0.855$ and $r=0.25, p=0.265)$. There was no relation between the ECD and the storage medium type $(p=0.109)$. The eye banks which did not calibrate their microscope reported higher ECDs than the others $(p=0.006)$. The use of the "borders method" seemed associated with higher ECDs ( $p=0.012$ ) but the use of the border method seemed more frequent when calibration was missing.

*The only eye bank to mark cells manually on a photographic print of the endothelium. The correcting factor $\times 1.5$ is due to this working method.

tThese two eye banks routinely use an image analyser, which necessitates either tracing the contours of cells or marking the cell centres. Routine performance of a second manual count authorised their inclusion in our study; however, the ECDs listed are those from the computer aided counts.

$\ddagger$ Storage at $4^{\circ} \mathrm{C}$ only.

$\mathrm{NA}=$ data not available. Other $=$ two personal unusual counting strategies .

(presence or lack of calibration, counting strategy) were tested using the non-parametric tests of Mann-Whitney for qualitative variables and the calculation of the Spearman correlation coefficient for quantitative data. The analysis was performed using the Social Package for the Statistical Sciences 11.0 software for Windows with $\mathrm{p}<0.05$ deemed significant.

\section{RESULTS}

All of the French eye banks in service in 2002 (22/22, 100\%) responded to the questionnaire. Twenty banks (20/22, 91\%) performed only manual cell counts under a light microscope. The corneas underwent osmotic preparation in $0.9 \% \mathrm{NaCl}$ until the mosaic reached optimal visibility, generally after 4 minutes of incubation $(13 / 22,59 \%)$, the time recommended by Delbosc when organ culture was introduced in France. ${ }^{7}$ Noticeably, one eye bank did not do so, and directly observed the endothelial monolayer. The eight others incubated the corneas for 2-3 minutes. One eye bank used $1.8 \%$ sucrose if mosaic visualisation proved difficult. At the time of the survey, only two eye banks (Table 1 , eye banks 11 and 17) were using an image analyser but in manual mode that required tracing of the cell borders or simply marking (clicking on) the cells on the computer screen. These two eye banks also performed manual counts and therefore responded to the questionnaire. One eye bank (Table 1, eye bank 8) counted cells from a photograph of the endothelium, marking the cells within a calibrated grid (Konan) overlay.

\section{Microscope, reticule, and calibration}

The eye banks used binocular, direct light microscopes. They were often simple models, and were manufactured by Leitz/ Leica $(9 / 22,41 \%)$, Nikon $(7 / 22,32 \%)$, Olympus $(5 / 22,23 \%)$, or Zeiss $(1 / 22,4 \%)$. In total, 12 different microscope bodies were used. With one exception ( $\times 20$ objective), all the eye banks used a $\times 10$ objective, most often $(9 / 22,41 \%)$ with a long or extra long working distance (so called "metallographic" objective). Four eye banks also used, where necessary, a $\times 20$ objective for closer observation of the endothelium. One eye bank used a $\times 2.5$ objective for global assessment of the corneal folds. All the eyepieces were $\times 10$, with one exception $(\times 12.5)$.

For 18 eye banks $(18 / 22,82 \%)$, the reticule was composed of a square grid divided into $10 \times 10$ identical square units, each $1 \mathrm{~mm}^{2}$. One eye bank used a reticule with a square grid divided into $5 \times 5$ units of $1 \mathrm{~mm}^{2}$; another used a square grid divided into $7 \times 7$ units of $1 \mathrm{~mm}^{2}$. Only one reticule was not a uniformly squared grid but a square with $7.2 \mathrm{~mm}$ sides, divided into four squares with, in their centre, a second square of $2.56 \mathrm{~mm}^{2}$ (Zeiss). All the reticules were positioned in one of the two eye pieces, except for one eye bank where it was placed in the microscope body. As mentioned above, one eye bank used a calibrated grid overlay directly placed on endothelial pictures.

Two thirds of the eye banks (14/22, 64\%) calibrated the microscope/reticule assembly, quarterly (two cases), half yearly (one case), annually (10 cases), or once in all (one case). Calibration (Fig l) was performed using a micrometric slide ( $1 \mathrm{~mm}$ or $2 \mathrm{~mm}$ long rule, with $0.1 \mathrm{~mm}$ divisions) (eight cases), a Thoma's grid $\left(1 \mathrm{~mm}^{2}\right.$ grid formed of multiples $2500 \mu \mathrm{m}^{2}$ surfaces) (three cases), or both (one case). Calibration was done by the eye bank (12 cases) or an external contractor (two cases). In six cases (Table 1, eye banks $8,9,12,13,17$, and 20) such calibration made it possible to determine a magnification correcting factor, which ranged from $\times 0.64$ to $\times 1.25$. The initial cell count in the reticule image was multiplied by this factor to obtain the ECD. In the other cases, calibration did not give rise to a 


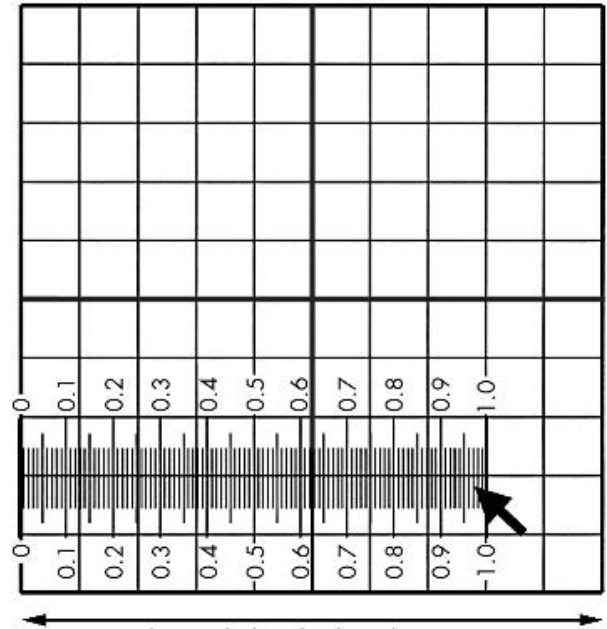

Real reticule border length $=10 \mathrm{~mm}$

Figure 1 Example of the procedure to calibrate a microscope fitted with an objective of $\times 10$ theoretical magnification. The reticule (a square grid of $100 \mathrm{~mm}^{2}$ real surface area) was placed in the eyepiece, with the engraved side in the plane of the diaphragm. A strip bearing a micrometric slide (arrowed) of $1 \mathrm{~mm}$ certified length was placed on the microscope's viewing stage, to measure the length of one side of the reticule border image. If the micrometer image did not coincide with the reticule border image, a magnification correcting factor was necessary. In this example, the length of the projected image of the $1 \mathrm{~mm}$ micrometer was not $10 \mathrm{~mm}$ as it should have been, but only $8 \mathrm{~mm}$. A real endothelial area of $1 \mathrm{~mm}^{2}$ thus projected in a reticule area of $8 \times 8$ $=64 \mathrm{~mm}^{2}$ (and not $100 \mathrm{~mm}^{2}$ ). It was consequently necessary to multiply the obtained cell count by $64 / 100$ to obtain the real ECD in cell $/ \mathrm{mm}^{2}$. The magnification factor was thus 0.64 in this example. If the micrometer image coincided with the reticule border image, no correcting factor was necessary.

correcting factor (so assumed to be $\times 1$ ). Eight eye banks $(8 / 22,36 \%)$ (Table 1 , eye banks $1,2,3,4,5,6,14$ and 15) did not calibrate their microscopes, and referred to the reticule manufacturers' data. It is notable that six of these reported the highest ECD values. Differences in the application of a correction factor were also identified. Two centres (Table 1, eye banks 9 and 20) used exactly the same microscope and reticule models yet applied different correcting factors despite each reporting that they performed their own calibration. Eye bank 9 applied a correcting factor of $\times 0.95$, compared to a correction of $\times 0.64$ used by eye bank 20 , and reported the higher ECD values. Likewise, two eye banks (Table 1, numbers 2 and 12) used similar microscopes and reticules, but only eye bank 12 applied a correcting factor $(\times 0.8)$. If this factor was applied to the mean ECD data of eye bank 2, the

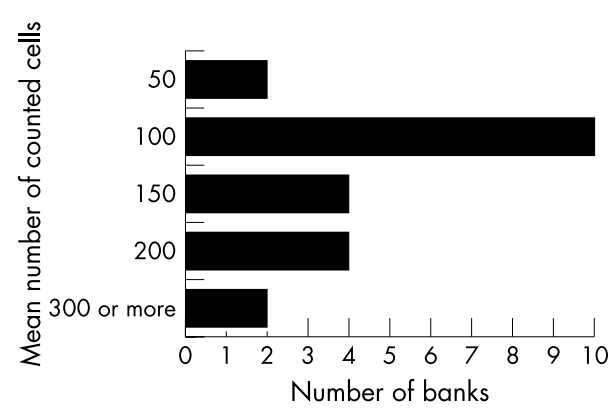

Figure 2 Mean number of endothelial cells taken into account for the endothelial assessment ( $n=22$ eye banks). More than half the eye banks $(12 / 22,55 \%)$ took into account only 50 or 100 cells. data from two eye banks became nearly similar. Likewise, eye banks 1 and 17 had similar microscopes. Eye bank 1 had not performed microscope calibration and reported higher ECD values, whereas eye bank 17, which used an image analyser, reported lower ECD values.

\section{Counting strategy}

The cell counting was most often (17/22, 77\%) carried out in the central $8 \mathrm{~mm}$ of the cornea, but was also carried out in the more peripheral cornea when the visibility of the central cells was inadequate. The number of non-adjacent endothelial zones examined was most often five $(9 / 22,41 \%)$, and varied from one to six. The cells were counted in one to 16 reticule units, most often in five units $(9 / 22,41 \%)$ and in mostly non-adjacent zones (17/22, 77\%). While almost half the eye banks $(10 / 22,45 \%)$ reported counting 100 cells for the ECD assessments, very different protocols were used at the other eye banks. In some, only 50 cells were counted, while others counted as many as 300 cells/endothelium (Fig 2). No association could however be detected between the number of cells counted and the resultant mean ECD reported by the eye bank (Spearman's non-parametric correlation coefficient, $r=-0.32, \mathrm{p}=0.198)$.

Most of the eye banks used one of two counting strategies. In most (17/22, 77\%), a "zone strategy" (Fig 3A) was used while the remainder $(3 / 22,14 \%)$ reported using a "border strategy" (Fig 3B). The three eye banks using the border strategy counted no more than 100 cells, and reported some of the highest ECD values (Table 1). Two eye banks (2/22, $9 \%$ ) used more unusual counting strategies. One (eye bank 4) counted the four borders of a square, while the other (eye bank 21) used the border method but with a reticule of a non-standard design.

\section{Technicians and counting exercise}

The 22 eye banks employed one to five technicians $($ total $=62$ ) with wide ranging experience levels. Thirteen (21\%) were "novice" (less than 100 counts), 29 (47\%) "experienced" (100 to 500 counts), and 20 (32\%) "expert" (more than 500 counts). Teams of two or three technicians were the most common (17/22, 77\%). None of the eye banks reported any double counting for verification of ECD reports. However, based on their performance in the standardised exercise (Fig 4), the analyses revealed that 12 eye banks (12/ $22,54 \%$ ) made one error. In seven cases, it concerned cell assignment at the intersection of two reticule borders (with the cell being counted in excess). In the other five cases, the errors involved misassignment (adding) of two or even three
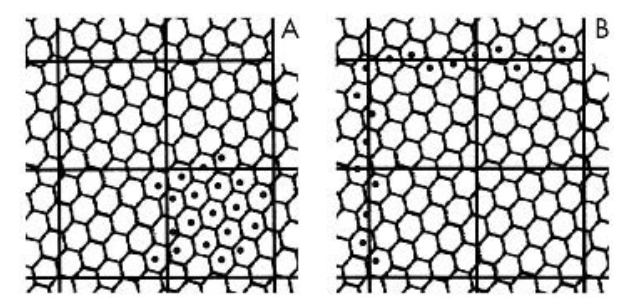

Figure 3 Illustration of the two endothelial counting strategies on a theoretical mosaic with uniform endothelial cell density (ECD), using a reticule with a $0.01 \mathrm{~mm}^{2}$ square surface area. The cells taken into account are marked. (A) "Zone strategy." Cells within the square unit are counted, and those touching two adjacent borders (in this case, the right hand side border and the bottom border) are excluded. This procedure is performed 1-10 times, depending on the eye bank. In this example, $\mathrm{ECD}=20 \times 100=2000 / \mathrm{mm}^{2}$. (B) "Border strategy." Cells touching two adjacent borders, two square units long. In this example, $\mathrm{ECD}=9 \times 9 \times 100 / 4=2025 \mathrm{cell} / \mathrm{mm}^{2}$. This example highlights a $1.25 \%$ difference between the two strategies. 

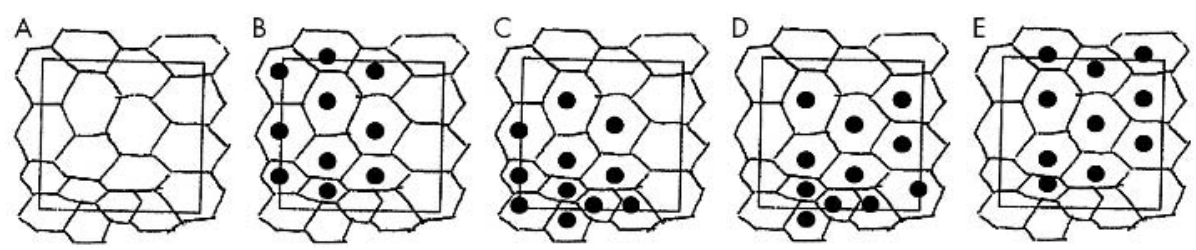

Figure 4 Proposed counting exercise (A). The endothelial cells usually taken into account had to be ticked. With the "zone strategy," the most common method, there were four acceptable answers depending on which borders were chosen. In the case of this non-uniform mosaic, the theoretical results were either 10 ( $B$ and $E$ ) or 11 (C and D) cells. This slight variation is an intrinsic drawback of the fixed frame method on a non-uniform mosaic. However, it is minimised by taking account of several zones.

cells to the count of 20, so substantially increasing the ECD estimate, in this particular example of an endothelium with a very low cell density.

\section{Presentation of ECD data and additional reporting of cell features}

Seven eye banks $(7 / 22,32 \%)$ did not assess or report the endothelial polymegethism along with the ECD. However, 10 eye banks $(10 / 22,45 \%)$ used a two point score for polymegethism (uniform or non-uniform mosaic) and four $(4 / 22,18 \%)$ a three point score (minor, moderate, or severe polymegethism). One eye bank failed to provide data on whether this was assessed or reported. Fourteen eye banks $(14 / 22,64 \%)$ did not provide any indication of cell pleomorphism ("hexagonality"), but four eye banks (4/22, $18 \%)$ used a two point score, and three $(3 / 22,14 \%)$ a three point score. Again, one eye bank did not provide data on this.

Half of the eye banks (11/22, 50\%) archived a photograph of the mosaic (a colour image in five cases) and three (3/22, $14 \%)$ provided the surgeon with a copy.

\section{DISCUSSION}

This study reveals an unacceptable diversity in the endothelial cell counting methods used in the eye banks of France. Such diversity in methods may well explain the marked differences in the mean ECD values reported by different eye banks for corneas sent for transplantation. Microscope calibration error seems to be the main factor, although it remains unclear as to who might be responsible for this and how often it might be checked. Analysis of the methods used also indicates that the cell counting strategy itself and technician skills are certainly not negligible causes of differences between eye banks. At a time when, in France and many European countries, legal requirements for donor selection and eye banks' compliance with standards are becoming very onerous, we felt it important to emphasise that the cornea quality control, which is based mainly on ECD measurement at delivery, is paradoxically subject to very little standardisation.

\section{Variability related to calibration of the light microscope}

The absence of or incorrect calibration of the microscope/ reticule assembly appears to be the main source of error. The counting reticules were most often of flat $1 \mathrm{~mm} \times 1 \mathrm{~mm}$ surfaces on which the endothelium image, magnified 10 times by the objective, was projected. This corresponds to a theoretical surface area of $0.1 \mathrm{~mm} \times 0.1 \mathrm{~mm}\left(0.01 \mathrm{~mm}^{2}\right)$. However, several characteristics of the eyepieces and microscope body may have distorted this theoretical magnification and made it necessary, in practice, to verify calibration case by case. There may also be a difference of a few per cent between the listed and real magnifications of standard objectives, as used by most of the eye banks. Advanced models of objectives, the correction of optical aberrations, the degree of numerical aperture, and the working distance may improve image resolution, but do not obviate the need for calibration. The microscope body and the binocular head are most often of average quality, and may include optical systems that significantly alter magnification. From personal experience we discovered that our microscope had a $\times 0.8$ lens, a detail not listed by the manufacturer. All of these potential imprecisions necessitate a careful calibration of such microscopes, in order to apply, where necessary, a magnification correcting factor. It is important to note that the absence of calibration (in eight eye banks) generally caused an overestimation of ECD; six of these eye banks consistently had some of the highest ECD values. Similarly, the examples of pairs of eye banks using the same equipment but only one of which applied a correcting factor are particularly revealing. Eight eye banks calibrated their microscopes themselves and decided against a correcting factor $(\times 1)$. The exact calibration procedure is not known, and unnoticed past errors may explain the variability between these eye banks. As an example, we are certain that one eye bank used an incorrect calibration factor because it was applied only to one dimension, and not to two $(\times 0.9$ instead of $\times 0.9^{2}$, which gives 0.81 ) (personal data).

The eye pieces themselves, however, cannot cause counting errors. They serve only to enlarge the image viewed by the technician, without altering its resolution or magnification. The reticule image, placed in the focal plane of the eyepiece diaphragm, thus enlarges as the endothelium image is enlarged, in constant ratio.

Our recent work on the examination of organ cultured endothelial mosaics using an image analyser ${ }^{8}$ has highlighted a systematic error common to all the eye banks using counting reticules $(18 / 22,86 \%)$. The reticules consist of uniform grid patterns printed on a glass disc, the lines of which are $2 \mu \mathrm{m}$ thick (source: Leica). The step, defined by the distance between the same borders of two successive lines (right hand borders, for example) is $1 \mathrm{~mm}$ but the internal distance between two lines is only $0.98 \mathrm{~mm}$. The surface area of each zone (in which the ECs are taken into account) is therefore not $1 \mathrm{~mm}^{2}$ but $0.9604 \mathrm{~mm}^{2}$. Although this is not relevant to the variability of eye banks' counting results, it consistently generates a $4 \%$ underestimation of ECD.

\section{Variability related to counting strategy}

Most banks use a zone strategy that is designed to assess cells within a fixed frame, in which the counting of cells should follow a fixed strategy-for example, excluding cells that contact only two of the borders of the counting grid. If the cell is considered to be a uniform entity, and if the sample size (cell count) is sufficient, then such a strategy can produce a very reproducible measure. ${ }^{9}$ The border strategy, adopted by three eye banks, consists of counting cells that touch two perpendicular borders of a grid. This method, however, lacks precision if the endothelial mosaic is not uniform and is perhaps associated with an overestimation of ECD. Whether this is routinely the case requires further study because there was a additional problem with microscope 
calibration in the eye banks using the border strategy. Regardless of the strategy adopted, it remains dependent on the skills of the technician and the time available. In routine practice, the technician would be expected to make decisions on cell contacts with the grid borders very quickly. This is difficult for cells with borders very close to the grid lines and/ or where the borders are rounded or of low contrast. The last feature is not uncommon following osmotic dilatation. In addition, the present survey of methods indicates that they are not always properly applied. As a result of these discrepancies, it was not possible to demonstrate any reasonable correlation between the eye bank estimated cell count (ECD) and the actual number of cells that were included in the count. Part of the reason for this relates to calibration problems. A related issue is the variance in ECD estimates in relation to the number of cells assessed. It has been reported that the predicted variance in ECD estimates decreases as the cell count increases. ${ }^{10}$ In these studies, it was demonstrated that even with cell counts approaching 100/ image, the uncertainty in ECD estimates could still be plus or minus $5 \%$, especially if the cell mosaic displayed polymegethism. In our later studies, ${ }^{8}$ we reported that the differences between manual counts and a computerised method were generally higher when only 50 cells were assessed, and we routinely prefer to assess some 300 cells from three distinct non-adjacent regions of the cell layer. ${ }^{8}$ This is readily possible with a computer based analysis but is impractical with a manual approach. Some of the eye banks reported assessments of multiple regions of the endothelium (for example, number 5) and it would be useful to further compare the outcome measures from verified manual counts and a computer analysis system to establish whether repeated assessments (for example, of two or more regions) will actually yield a superior outcome measure. It is important that these multiple regions are chosen from within the central region of the cornea (that is, within the $8 \mathrm{~mm}$ diameter zone usually used for a graft). Attempts to assess ECD based on counts from peripheral zones, as reported by one eye bank in five, may result in substantial overestimation of ECD since the cell count in the extreme periphery of the cornea can be expected to be rather higher. ${ }^{11}$

\section{Possible variability related to technician skills}

In France, it is considered very important to provide extensive training to technicians who will be employed in eye banks to provide endothelial cell counts. The process begins with theoretical training provided by the national transplantation agency (Etablissement Français des Greffes), and is completed with practical coaching by fellow technicians in the same eye bank. Counting skills are therefore liable to drift over time. The large number of technicians is a major factor in variability. The 62 technicians of the 22 eye banks evaluated about 6000 corneal endothelia yearly, a mean of about two corneas per week per technician. The need for continuous grafting activity requires each eye bank to have at least two technicians; they are often part time, reducing each one's experience. Moreover, it should be noted that no count was routinely verified by a second technician, and that the post-count documents did not usually allow the surgeon to subsequently verify the count quality.

There are a number of practical issues that need to be addressed in relation to the reliability of viewing and counting cells in organ cultured endothelia. Visibility of the intercellular spaces is often mediocre; focusing on a wide endothelial surface is difficult because of the posterior folds; and the count must be done promptly to limit the time the cornea spends out of its storage medium. Sperling, ${ }^{12}{ }^{13}$ after staining EC membranes with alizarin red, which provides excellent experimental conditions for viewing cell contours, showed that manual evaluation of ECD was accurate only to plus or minus $5 \%$. In the absence of vital staining of cell membranes, mosaic visibility can be increased and count accuracy significantly increased by allowing optimum time interval, usually about 4 minutes, using sucrose, and examining more endothelial zones. Lastly, the time factor can be eliminated by "deferred" counting from a photograph (eye bank 8 ) or by using an image analysis system (eye banks 11 and 17).

\section{Sources of variability related to type of data acquisition and presentation}

Objective cell analysis based on measures of cell areas, the variance in cell areas, and quantification of pleomorphism was only available in a few eye banks despite its importance in tissue selection before grafting. ${ }^{14}$ While some form of subjective grading of endothelial cell polymegethism and pleomorphism was carried out by over half of the laboratories, such a method does not provide adequate information for later use by the surgeon. This is because the postoperative assessments will more likely use in vivo specular microscopy that, with some form of analysis software, will provide an objective cell analysis output. Lastly, from the perspective of being able to relate eye bank data presentation (on cell counts or grading) to postoperative outcomes, it has to be noted that only half of the eye banks retained an archive of endothelial images. This is needed to allow for tracing of material and to develop better quality control procedures.

\section{CONCLUSIONS}

The present study has identified some substantial problems with the current methods of manual counts of endothelial cells by eye banks. Some of these should be readily rectifiable-for example, errors of calibration applied to the microscope images. However, the present study hopefully also serves to highlight the inherent variability in the manual count method such that the current criteria often used for acceptability of donor corneas have to be questioned. A central cell count of above 2000 cells $/ \mathrm{mm}^{2}$ is often used as a cut off for selection of donor corneas yet the variability in the manual counting methods may be unable to adequately distinguish between endothelia that are close to this value. If an acceptable variance in the outcome is plus or minus 5\%, then it is likely that some eye banks discard too many corneas while others deliver corneas that, in reality, have too low a cell count. First of all, we would like this work to be a strong incitement to standardise the count process in all eye banks. Secondly, based on our previous studies, ${ }^{8}{ }^{15}$ we suggest that eye banks should be upgraded to routinely be able to carry out objective cell analysis based on semiautomated measures of cell areas, the variance in cell areas, and quantification of pleomorphism. Such a change will be expensive but will provide a much better quality control for donor corneas.

\section{ACKNOWLEDGEMENTS}

The authors wish to thank the scientific managers and technicians of the 22 French eye banks who responded to the questionnaire.

\section{Authors' affiliations}

G Thuret, C Manissolle, J Maugery, L Campos-Guyotat, P Gain, "Cell Survival and Adhesion in Cancers and Graffs" Research Group, EA 3063, Faculty of Medicine, and Ophthalmology Department, Bellevue University Hospital, Saint-Etienne, France

S Acquart, J-C Le Petit, Cornea Bank, Etablissement Français du Sang Auvergne-Loire, Saint-Etienne, France

M J Doughty, Department of Vision Sciences, Glasgow-Caledonian University, Glasgow, UK

Proprietary interest: None. 
Presented in part at the annual meetings of the Sociéte Française d'Ophtalmologie, in Paris, May 2002, and of the European Eye Bank Association, in Brussels, January 2003.

\section{REFERENCES}

1 EEBA. European Eye Bank Association Directory. 10th ed, 2002

2 Pels E, Schuchard Y. Organ-culture preservation of human corneas. Doc Ophthalmol 1983;56:147-53.

3 De Boroviczeny CG. On the standardization of the blood cell counts. Bibl Haematol 1966;24:2-31.

4 French directory of corneal storage centres. 7th ed. Besançon, 1999.

5 French directory of corneal storage centres. 8th ed. Besançon, 2000.

6 French directory of corneal storage centres. 9th ed. Besancon, 2001.

7 Delbosc B, Borderie V. Methods of preservation of the human corneas. J Fr Ophtalmol 1997; 20:221-40.

8 Gain P, Thuret G, Kodiikian L, et al. Automated tri-image analysis of stored corneal endothelium. Br J Ophthalmol 2002;86:801-8.
9 Gundersen HJ. Estimators of the number of objects per area unbiased by edge effects. Microsc Acta 1978;81:107-17.

10 Doughty MJ, Muller A, Zaman ML. Assessment of the reliability of human corneal endothelial cell-density estimates using a noncontact specular microscope. Cornea 2000;19:148-58.

11 Schimmelpfennig BH. Direct and indirect determination of nonuniform cell density distribution in human corneal endothelium. Invest Ophthalmol Vis Sci 1984;25:223-9.

12 Sperling S, Gundersen HJ. The precision of unbiased estimates of numerical density of endothelial cells in donor cornea. Acta Ophthalmol (Copenh) 1978:56:793-802.

13 Sperling S. Indirect evaluation of corneal endothelial cell density. Acta Ophthalmol (Copenh) 1978;56:445-54.

14 Borderie VM, Scheer S, Touzeau O, et al. Donor organ cultured corneal tissue selection before penetrating keratoplasty. $\mathrm{Br} J$ Ophthalmol 1998;82:382-8.

15 Gain $\mathbf{P}$, Thuret G, Chiquet $C$, et al. Automated analyser of organ cultured corneal endothelial mosaic. J Fr Ophtalmol 2002;25:462-72.

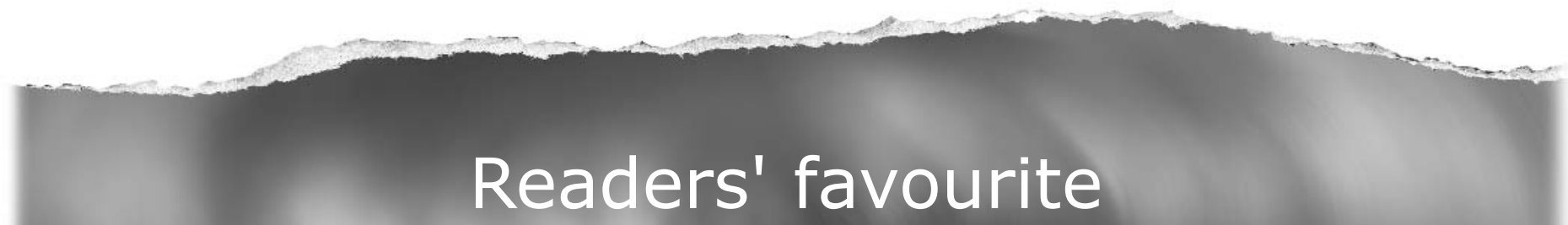

\section{Top 10}

Click on the "Top 10" button on the homepage

to see which are the best read articles each month

www.bjophthalmol.com 